\title{
Les jeux de l'injure dans Henry IV
}

Nathalie Vienne-Guerrin

\section{Q OpenEdition \\ Journals}

\section{Édition électronique}

URL : http://journals.openedition.org/shakespeare/713

DOI : 10.4000/shakespeare.713

ISSN : 2271-6424

Éditeur

Société Française Shakespeare

\section{Édition imprimée}

Date de publication : 1 novembre 2005

Pagination : 185-199

ISBN : 2-9521475-2-3

\section{Référence électronique}

Nathalie Vienne-Guerrin, "Les jeux de l'injure dans Henry IV », Actes des congrès de la Société française Shakespeare [En ligne], 23 | 2005, mis en ligne le 01 janvier 2007, consulté le 20 avril 2019. URL : http:// journals.openedition.org/shakespeare/713; DOI : 10.4000/shakespeare.713 


\section{Shakespeare et le jeu}

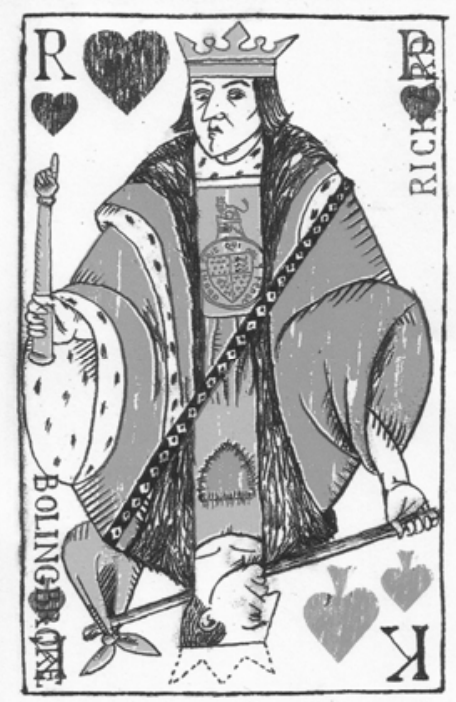

actes du Congrès organisé par la

SOCIÉTÉ FRANÇAISE SHAKESPEARE les 10, 11 et 12 mars 2005

textes réunis par Pierre KAPITANIAK sous la direction de Yves PEYRÉ 


\title{
COMITÉ SCIENTIFIQUE :
}

\author{
Margaret Jones-Davies \\ Gisèle Venet \\ Jean-Marie Maguin \\ Yves Peyré \\ François Laroque \\ Pierre Kapitaniak
}

COUVERTURE :

Edouard Lekston, King and Kusin, 2005

edlek@free.fr

conception graphique et logo

Pierre Kapitaniak

(C) 2005 Société Française Shakespeare

Institut du Monde Anglophone

Université de Paris III - Sorbonne Nouvelle

http://recherche.univ-montp3.fr/SFS/

5 rue de l'École de Médecine

75006 Paris

ISBN 2-9521475-2-3

Tous droits de traduction, de reproduction et d'adaptation réservés pour tous les pays 


\title{
LES JEUX DE L'INJURE DANS HENRY IV
}

\author{
Nathalie VIENNE-GUERRIN
}

\begin{abstract}
II s'agit d'analyser les mécanismes ludiques qui président aux « joutes de jactance » qui sont si caractéristiques d'Henry IV et à l'aune desquelles on peut mesurer l'ambiguïté et l'évolution de la relation entre Falstaff et Hal / Henry. Nous examinons comment ces tournois d'injures se manifestent sur la scène shakespearienne. II ne s'agit pas d'une étude rhétorique et lexicale de l'injure mais de l'examen d'un rituel verbal paradoxal au cours duquel on s'insulte sans s'injurier, au cours duquel (pour reprendre la terminologie d'Évelyne Larguèche) l'injure n'a pas, ou est censée ne pas avoir, "l'effet injure ». Nous explorons les règles propres à ce jeu de langue et nous intéressons notamment aux glissements de l'injure du mode ludique au mode sérieux. Après avoir délimité les modalités du jeu de l'injure telles qu'elles apparaissent dans la première partie d'Henry IV, nous nous attachons à montrer comment ce jeu s'avère dangereux avant d'être finalement déclaré hors-jeu dans la deuxième partie.
\end{abstract}

This paper proposes to analyse the ludic mechanisms on which the flyting scenes of Henry IV are based. We show how these flyting scenes reveal the ambiguity and evolution of the relationship between Falstaff and the Prince / King. We examine how these verbal tournaments take place on the Shakespearean stage. This paper does not offer a rhetorical and lexical analysis of the play's insults but explores a paradoxical verbal ritual in which abuse is - or is supposed to be - "no abuse." We analyse the rules on which this verbal game is based and notably examine how the boundaries between ludic flyting and serious flyting are blurred in the two plays. After describing the rules of this comic verbal activity as they are dramatized in 1 Henry IV, we show how this game proves dangerous and how the King decides to prohibit it in 2 Henry IV.



e me souviens - et je ne suis probablement pas la seule à m'en souvenir - avoir commencé la soutenance de ma thèse consacrée à l'injure shakespearienne en débitant à mon jury un énorme chapelet d'insultes. Ces injures monumentales, je les avais bien sûr mises entre guillemets ; je les avais empruntées à Falstaff et à Hal. Je les avais extraites du cœur d'Henry IV (première partie), de l'acte II scène iv, pour les inscrire, à l'occasion d'un rituel des plus sérieux, dans une démarche ludique. Ce jour-là ces injures n'ont pas eu, je l'espère, «l'effet injure » si bien décrit par Évelyne Larguèche ${ }^{1}$. Ce jour-là, le jury a compris, je l'espère, que c'était « pour rire ». Il y avait dans ces bordées d'injures la manifestation de ce que j'avais voulu montrer dans ma thèse : un chapelet d'insultes n'est pas forcément injurieux; une injure n'a pas toujours «l'effet injure »; le mot d'injure ne fait pas forcément mal, mais peut être divertissant, peut être « excellent sport »

\footnotetext{
${ }^{1}$ Évelyne Larguèche, L'Effet injure. De la pragmatique à la psychanalyse, coll. «Voix nouvelles en psychanalyse ", Paris, PUF, 1983.
} 
(1H4, II.iv.380 2 ), comme le dit l'hôtesse en assistant aux joutes verbales de Hal et de Falstaff. Tout dépend de la situation d'énonciation. S'il y avait eu dans mon jury un fort gros ou un fort maigre, ces chapelets débités sur le mode ludique auraient-ils été reçus de la même oreille? J'en doute. En matière d'injure, la réception compte autant que l'émission. Ce jeu de l'injure se joue à deux. Pour qu'il y ait jeu d'injure, il faut qu'un contrat soit passé entre une langue et une oreille. En insultant le jury, même avec des guillemets, j'avais pris un risque; j'avais pris le risque que les destinataires le «prennent mal ». Je me demande toujours d'ailleurs si ces injures d'emprunt ont bien été inoffensives, ont bien été "no abuse » $\left(2 \mathrm{H} 4\right.$, II.iv. $\left.310^{3}\right)$ pour ces cinq éminents chercheurs. Après tout, peut-être certains ont-ils considéré que ce n'était ni le lieu ni l'endroit pour « jouer » à cela, que j'étais là, comme le bon gros Falstaff sur le champ de bataille, en train de me tromper de terrain de jeu. Ce jeu n'avait peut-être rien à faire dans cette bagarre qu'est une soutenance de thèse. Si ces mots n'avaient pas l'effet injure, peut-être l'acte de langage en lui-même avait-il semblé déplacé, hors de propos ; peut être que le fait même de jouer, ou le fait de ne pas jouer le jeu attendu, avait eu l'effet injure. Le jury a-t-il alors pensé « excellent sport » comme l'hôtesse, ou bien s'est-il dit, comme Hal lorsqu'il reproche à Falstaff son inconséquence frivole, « What, is it a time to jest and dally now ? » (1H4, v.iii.57).

Ce préambule autobiographique aura, je l'espère, révélé tout le « jeu » - dans le sens dérridéen du terme 4 - qu'il y a dans ce jeu-là, toute la complexité des jeux de l'injure et notamment les « frottements » possibles entre l'injure ludique et l’injure sérieuse, ou, pour ne pas employer cette antithèse (ludique / sérieux) invalidée par Johan Huizinga ${ }^{5}$, les glissements probables de l'injure sans effet à

\footnotetext{
${ }^{2}$ William Shakespeare, King Henry IV. Part 1, éd. David Scott Kastan, The Arden Shakespeare, Third Series, 2002. Toutes les citations suivantes renverront à cette édition.

3 William Shakespeare, King Henry IV. Part 2, éd. A. R. Humphreys, The Arden Shakespeare, Londres et New York, Routledge, 1967. Toutes les citations suivantes renverront à cette édition.

4 Pour un survol des définitions du terme « jeu », voir Anna K. Nardo, The Ludic Self in Seventeenth-Century English Literature, New York, State University of New York Press, 1991, chap. 1, p. 1-13.

5 Johan Huizinga, Homo Ludens. Essai sur la fonction sociale du jeu, Paris, Gallimard, 1951, p. 42-56.
} 
«l'effet injure ». Notre propos est de montrer comment, dans les deux parties d'Henry IV, Shakespeare exploite ces jeux de l'injure non seulement afin de construire un univers carnavalesque des plus Bakhtiniens ${ }^{6}$ mais aussi pour suggérer toute la complexité et la fragilité des rapports entre Hal et Falstaff. Dans un article intitulé « No abuse : The Prince and Falstaff in the Tavern Scenes of Henry IV", J. McLaverty note que Hal et Falstaff ne semblent jamais aussi proches l'un de l'autre que lorsqu'ils s'insultent ${ }^{7}$. Selon lui, ce que les deux personnages perdent dans la deuxième partie, c'est précisément cette intimité qui rend les injures inoffensives («the intimacy which makes abuse innocuous $\left.{ }^{8} »\right)$. Nous nous proposons ici de faire apparaître les mécanismes ludiques qui président à ces échanges d'injures, à ces épisodes de «flyting 9 ", à ces « joutes de jactance » comme les appelle Huizinga dans Homo Ludens ${ }^{10}$, joutes à l'aune desquelles on peut mesurer non seulement l'évolution mais aussi l'ambiguité de la relation

\footnotetext{
${ }^{6}$ Mikhaïl Bakhtine, L'Euvre de François Rabelais et la culture populaire au Moyen Âge et sous la Renaissance, Paris, Édition Gallimard, 1970, p. 428-32. Cette idée du double ton du mot, de la fusion de l'éloge et de l'injure, est également développée p. 25, 167 et sqq., 248-9, $407,412-17,454$ et sqq.

7 Shakespeare Survey, $\mathrm{n}^{\circ} 34,1981$, p. 105-110 : « it is when the prince and Falstaff are most in conflict, when they are exchanging abuse, that we are most aware of how close they are and how much they share. In their efforts to cap insults they reveal their familiarity with, and dependence on, one another » (p. 106-107).

8 Op. cit., p. 105.

${ }^{9} \mathrm{Ce}$ terme est devenu tellement polysémique que je me contenterai pour l'heure de l'employer dans son sens le plus simple d'échange d'injures ritualisé. L'OED retient essentiellement l'origine écossaise du «flyting » en définissant le terme comme suit: " Poetical invective ; chiefly, a kind of contest practised by the Scottish poets of the 16th century, in which two persons assailed each other alternately with tirades of abusive verse ». Pour une analyse plus poussée des diverses conceptions du «flyting ", voir notamment, Priscilla Bawcutt, « The Art of Flyting », Scottish Literary Journal, vol. x, $\mathrm{n}^{\circ}$ 2, 1983, p. 5-24; Carol J. Clover, "The Germanic Context of the Unferth Episode", Speculum, $\mathrm{n}^{\circ}$ 55, 3, 1980, p. 444-68 ; Robert C. Elliott, The Power of Satire : Magic, Ritual, Art, Princeton, New Jersey, Princeton University Press, 1960, p. 70 et sqq. ; Margaret Galway, "Flyting in Shakspere's Comedies », The Shakespeare Association Bulletin, $\mathrm{n}^{\circ} \mathrm{x}$, iv, 1935, p. 183-91; Johan Huizinga, op, cit., p. 119-20 ; William Labov, Language in The Inner City: studies in the Black English Vernacular, Philadelphie, University of Pennsylvania Press, 1972 ; Ward Parks, Verbal Dueling in Heroic Narrative. The Homeric \& Old English Traditions, Princeton, Princeton University Press, 1990 ; Frank J. Warnke, "Amorous Agon, Erotic Flyting : Some Play-Motifs in the Literature of Love », in Auctor Ludens, Essays on play in Literature, éd. G. Guinness et A. Hurley, Philadelphie, John Benjamins, 1986, p. 99-112 (Collection Cultura Ludens). Trois catégories essentielles de "flyting » ressortent de tous ces textes : le «flyting » ludique, le «flyting " héroïque et le «flyting » érotique.

10 Op. cit., p. 114.
} 
entre Falstaff et Hal. Il ne s'agira pas d'une étude rhétorique et lexicale de l'injure ${ }^{11}$, mais de l'examen d'une activité verbale paradoxale, au cours de laquelle on s'insulte sans s'injurier, au cours de laquelle l'injure n'a pas - ou est censée ne pas avoir - «l'effet injure». En d'autres termes, nous ne nous attacherons pas ici au contenu des injures si nombreuses dans Henry IV mais à l'acte de langage ( « speech act ») ludique lui-même ${ }^{12}$. Après avoir délimité les modalités du jeu de l'injure telles qu'elles apparaissent dans la première partie d'Henry IV, nous nous attacherons à montrer comment ce jeu s'avère dangereux avant d'être finalement déclaré hors-jeu dans la deuxième partie.

\section{«Excellent sport » $\left(1 \mathrm{H}_{4}\right.$, II.iv.380)}

« Well said, Hal! To it, Hal ! Nay, you shall find no boy's play here, I can tell you» (1H4, v.iv.74-75): voilà en quels termes Falstaff encourage son petit Hal lorsque celui-ci affronte enfin Hotspur sur le champ de bataille. On peut entendre dans l'échange qui précède ce combat (1H4, v.iv.58-73) un épisode de ce que Ward Parks appelle le " flyting " héroïque ${ }^{13}$, cet échange d'injures et de rodomontades entre deux adversaires, qui constitue un rite de passage des mots aux actes et par lequel les deux parties signent en quelque sorte un pacte, un contrat d'agression. Le commentaire de Falstaff signale que l'on est passé du combat verbal ludique et infantile («boy's play») qui caractérisait l'espace de la taverne au duel verbal héroïque qui mène à la mort. Contrairement à l'échange hérö̈que décrit par Ward Parks, l'injure ludique se doit, pour rester un jeu, de ne pas mener aux actes. La règle d'or du «flyting » ludique, c'est que les injures qui le constituent n'aient pas «l'effet injure ». Or ce sont bien ces injures sans effet qui semblent régner dans l'univers de la taverne où Shakespeare donne à entendre ce que Bakhtine appelle le « double ton

\footnotetext{
${ }^{11}$ Pour une étude rhétorique de la question, voir Nathalie Vienne-Guerrin, «L'anatomie de l'insulte dans 1 Henry IV », Bulletin de la Société de Stylistique Anglaise, ${ }^{\circ}$ 17, 1996, p. 2135 .

12 Pour un survol du «flyting » comme acte de langage, voir Andreas H. Jucker et Irma Taavitsainen, « Diachronic Speech act analysis. Insults from flyting to flaming », Journal of Historical Pragmatics, vol. I (1), 2000, p. 67-95.

13 Voir Ward Parks, op. cit.
} 
du mot » qui efface les limites entre terme d'injure et terme d'affection ${ }^{14}$. " Thou hast the most unsavoury similes and art indeed the most comparative, rascalliest, sweet young prince. " (1H4, I.ii.768) : dans le monde festif d'Henry IV, les injures, pour être amères ( « unsavoury »), n'en sont pas moins douces («sweet») et l'énorme panse de Falstaff ne semble avoir aucun mal à les digérer. Dans Henry $I V$, l'injure est jeu de langue et son espace est la taverne.

Après avoir installé l'injure comme mode d'adresse privilégié au cours des deux premiers actes, Shakespeare nous offre un véritable combat d'injures comique au cours de l'acte II, scène iv :

PRINCE. I'll be no longer guilty of this sin. This sanguine coward, this bed-presser, this horse-back-breaker, this huge hill of flesh -

FALSTAFF. 'Sblood, you starveling, you eel-skin, you dried neat's tongue, you bull's pizzle, you stock-fish ! O, for breath to utter what is like thee ! You tailor's yard, you sheath, you bow-case, you vile standing tuck-

PRINCE. Well, breathe awhile and then to it again, and when thou hast tired thyself in base comparisons, hear me speak but this.

(1H4, II.iv.235-44)

Ce que l'on a pu lire comme la querelle des Gros et des Maigres ou le combat de Carnaval et de Carême ${ }^{15}$ est une compétition d'injures ( «̂̂gon »), un combat de boxe verbal qui laisse Falstaff à bout de souffle, le fait probablement suer à grosses gouttes et semble propre à donner le vertige («Ilinx »). Tout comme les jeux de langage décrits par Claude Gilbert-Dubois dans Mots et Règles, Jeux et Délires ${ }^{16}$, le jeu de l'injure repose sur une virtuosité, une acrobatie langagière qui produit des « monstres linguistiques ${ }^{17}$ » et confine à l'ivresse, voire au délire, au vertige ${ }^{18}$. Il n'est pas fortuit que Falstaff, cet acrobate du

\footnotetext{
${ }^{14}$ La description que Falstaff fait de Bardolph et de son nez est particulièrement emblématique de cette fusion de la louange et de l'injure. Voir notamment « $\mathrm{O}$, thou art a perpetual triumph, an everlasting bonfire-light ! » (1H4, III.iii.4O-1).

${ }^{15}$ Voir notamment l'article de François Laroque «Shakespeare's 'Battle of Carnival and Lent'. The Falstaff Scenes Reconsidered ( 1 \& 2 Henry IV) » dans Shakespeare and Carnival. After Bakhtin, éd. Ronald Knowles, Houndmills, Basingstoke, Macmillan, 1998. Voir aussi Nathalie Vienne-Guerrin, op. cit.

16 Claude Gilbert-Dubois, Mots et règles, jeux et délires. Études sur lïmaginaire verbal au XVIe siècle, Caen, Paradigme, 1992.

17 Op. cit., p. 122.

${ }^{18}$ Voir le chapitre intitulé "L’invention Littéraire et les Jeux du Langage: jeux de nombres, jeux de sons, jeux de sens », op. cit, p. 121-45.
} 
langage, se compare à Hercule juste après avoir accompli son exploit langagier le plus remarquable, son entourloupette la plus mémorable :

PoINs. Come, let's hear, Jack. What trick hast thou now ?

FALSTAFF. By the Lord, I knew ye as well as he that made ye. Why, hear you, my masters : was it for me to kill the heir apparent ? Should I turn upon the true prince? Why, thou knowest I am as valiant as Hercules, but beware instinct.

(1H4, II.iv.258-63)

Falstaff est bien un Hercule. Il est ce dieu du langage que nous décrit Claude-Gilbert Dubois :

dieu du langage, dont le carquois porte-flèches et les flèches porteplumes signifient le jeu élocutoire. Le jeu d'archer d'Hercule désigne le fonctionnement de l'énonciation, qui consiste à choisir, dans le carquois du lexique, la flèche messagère appropriée - le mot propre - pour l'adapter à l'arc de la phrase, lui insuffler la force cinétique du verbe, et la projeter vers son destinataire. La signification linguistique du mythe est évidente, et développe une autre représentation emblématique d'Hercule, plus courante, celle de l'auditoire enchaîné par les oreilles à sa bouche, qui veut dire à la fois le circuit qui s'instaure dans le fonctionnement du message, et la fascination exercée par les mots habilement proférés. On sait que cette fascination peut revêtir un sens négatif et signifier les duperies de cette puissance trompeuse qu'est l'éloquence habile à faire passer n'importe quel mensonge ${ }^{19}$.

On croirait lire ici une description de Falstaff. Hal est, quant à lui, comparé à Mercure ("feathered Mercury », $1 H 4$, Iv.i.105), Hermès, autre dieu du langage, jongleur de mots dont les paroles s'envolent ${ }^{20}$. « I'll so offend to make offence a skill » nous dit Hal (I.ii.206). Le jeu dont nous parlons ici est un « jocus » (« jeu en paroles, plaisanterie»), terme dont les dérivés «jogler», « jongler» («juggler») sont euxmêmes reliés à l'ancien français « jangler » («jangling ») qui signifie "plaisanter, hâbler, médire ${ }^{21}$ ». Ces mots renvoient bien à ces acrobaties verbales si caractéristiques d'Henry IV. Hal et Falstaff sont des « jongleurs », terme issu du latin « joculator» qui signifie « rieur,

\footnotetext{
${ }^{19}$ Claude-Gilbert Dubois, "'La parole est pennigère'. Une interprétation du rôle de Mercure dans la 'Fable d'Io' (sur un texte de Geoffroy Tory) », op. cit., p. 27-36, p. 29. Pour une analyse des allusions au mythe d'Hercule dans l'œuvre de Shakespeare, voir M. T. Jones-Davies, "Shakespeare et le Mythe d'Hercule», Les Mythes Poétiques au Temps de la Renaissance, éd. M. T. Jones Davies, Paris, Jean Touzot, 1985, p. 127-46.

${ }^{20}$ Op. cit., p. 28-9.

${ }^{21}$ Dictionnaire Historique de la Langue Française, sous la direction d'Alain Rey, Paris, Dictionnaires le Robert, 1992.
} 
bon plaisant, railleur ${ }^{22}$ ». Dans ce monde de la taverne, les exploits sont verbaux, les hauts faits sont des dires, et les « gestes » des farces («jests »). Le duel d'injures entre Hal et Falstaff est annoncé dès le premier acte, lorsque Poins propose à Hal de mettre en œuvre le traquenard de Gad's Hill :

The virtue of this jest will be the incomprehensible lies that this same fat rogue will tell us when we meet at supper. [...] and in the reproof of this lives the jest.

(1H4, I.ii.176-80)

Ce duel entre le Prince et Falstaff est programmé. En tendant ce guetapens, c'est bien un jeu de langue que Poins et Hal sont en train de construire. Derrière le démenti (« reproof ») que le Prince va opposer aux mensonges de Falstaff, derrière la guerre des langues qui nous est annoncée, on entend déjà les injures qui se préparent. Le mot « reproof» signifie d'ailleurs également «insulte» selon l'OED. Falstaff lui-même instaure dès le début une règle dans ce jeu, en jurant de dire la vérité en ces termes : «I tell thee what, Hal, if I tell thee a lie, spit in my face, call me horse » $\left(1 H 4\right.$, II.iv.186-7) ${ }^{23}$. L'injure est la sanction comique que Falstaff propose d'infliger au menteur.

Tout le début de la pièce vise à construire cet échange d'injures, et à faire de ce choc des Titans un sommet dramatique. Le mot se fait ici spectacle. Contrairement à Hotspur qui peste en solitaire et insulte en pensée, notamment lorsqu'il s'imagine dresser un sansonnet («starling ») pour lui apprendre à outrager le Roi à coup de « Mortimer » (1H4, I.iii.222-4), les partenaires de jeu deviennent des créateurs qui offrent leurs trouvailles à la contemplation ${ }^{24}$. L'épisode de Francis et de ses inlassables « anon » (1H4, II.iv.26-79) ne fait que mieux ressortir toute la créativité du jeu d'injure. Shakespeare met en oeuvre un contraste flagrant entre ces deux types de guet-apens verbaux. Ce jeu de l'injure est en effet des plus spectaculaires et réclame le public de la taverne. Dans Chimes at Midnight (1966),

\footnotetext{
22 Ibid.

23 Ceci est une structure récurrente dans la pièce.

24 Pour des réflexions sur les exhibitions poétiques, voir le chapitre de Claude-Gilbert Dubois " 'Vice de innovation' et 'Escumeurs de latin'. Quelques aspects du mélange des langues dans ses rapports avec la création littéraire en France au XVI ${ }^{\mathrm{e}}$, op. cit., p. 61-74, p. 63 .
} 
Orson Welles crée, grâce aux décors en bois, un espace chaleureux et ludique qui contraste avec la froideur de la cour suggérée par la pierre. La taverne y apparaît comme un terrain de jeu où Hal et Falstaff se donnent en spectacle et délimitent un véritable espace scénique. La pièce dans la pièce que nous jouent les deux hommes rend plus explicite encore cette dimension spectaculaire et re/récréative du jeu d'injure. Les deux partenaires se font alors littéralement une "scène " au cours de laquelle injure et louange semblent être les deux facettes d'un même objet :

[...] that trunk of humours, that bolting-hutch of beastliness, that swollen parcel of dropsies, that huge bombard of sack, that stuffed cloak-bag of guts, that roasted Manningtree ox with the pudding in his belly, that reverend Vice, that grey Iniquity, that father Ruffian, that Vanity in years [...].

(1H4, II.iv.437-42)

[...] sweet Jack Falstaff, kind Jack Falstaff, true Jack Falstaff, valiant Jack Falstaff [...].

(1H4, II.iv.463-5)

On assiste ici au combat ludique de l'injure et de la louange. Falstaff ne répond pas à l'injure par l'injure mais lui oppose un autoportrait des plus flatteurs. Même si l'on n'a pas affaire à un échange d'injures, l'injure est intégrée à un double jeu, de langue et de rôle. Elle est à la fois jeu d'imitation et de compétition.

À l'acte III, scène ii, Shakespeare met en scène un autre type de jeu au cours duquel Hal et Falstaff s'associent pour malmener l'hôtesse. Après l'avoir traitée de "woman » ( "Go to, you are a woman, go », III.iii.6o), Falstaff fait de l'hôtesse une espèce de «machin chose » («thing») avant d'en faire une bête («beast») puis une loutre (« otter »):

FALSTAFF. Go, you thing, go !

HOSTESS. Say, what thing, what thing ?

FALSTAFF. What thing ? Why, a thing to thank God on.

HOSTESS. I am no thing to thank God on. I would thou shouldst know it. I am an honest man's wife, and, setting thy knighthood aside, thou art a knave to call me so.

FALSTAFF. Setting thy womanhood aside, thou art a beast to say otherwise.

HOSTESS. Say, what beast, thou knave thou.

FALSTAFF. What beast ? Why, an otter.

PRINCE. An otter, Sir John? Why an otter ? 
FALSTAFF. Why ? She's neither fish nor flesh ; a man knows not where to have her.

HOSTESS. Thou art an unjust man in saying so. Thou or any man knows where to have me, thou knave thou.

PRINCE. Thou sayst true, hostess, and he slanders thee most grossly. (III.iii.115-31)

L’injure « otter » crée un véritable effet de surprise : " why an otter ? » demande Hal. Il semble que Falstaff ait tiré ici un mot au hasard, dont l'incongruité est signalée par la question de Hal. On retrouve le même effet de surprise dans 2 Henry IV où le Page, qui a été à bonne école 25 lance à Bardolph un «you rascally Althaea's dream » que Hal lui demande d'expliciter («Instruct us, boy; what dream, boy? ») avant de l'homologuer en ces termes: «A crown's-worth of good interpretation! » (2H4, II.ii.83-7).

Dans Les Jeux et les hom mes, Roger Caillois ${ }^{26}$ distingue quatre sortes de jeu fondées respectivement sur la compétition («Ầgon»), le hasard («Alea »), le simulacre (« Mimicry »), le vertige (« Ilinx »). Le jeu de l'injure tel que nous venons de le décrire semble réunir ces quatre dominantes. Mais la «scène de la loutre » est également emblématique de l'équilibre instable sur lequel reposent les jeux injurieux. Si au cours de cette scène Hal et Falstaff s'amusent à insulter l'hôtesse, si pour eux l'injure est un jeu, l'hôtesse quant à elle semble bien ressentir "l'effet injure ». Le fait qu'elle veuille comprendre le contenu des insultes qui lui sont lancées signale qu'elle ne joue pas, que pour elle les mots doivent vouloir dire quelque chose et ne peuvent être de simples projectiles lancés au hasard. Cette scène montre également combien l'effet perlocutoire de l'injure est affaire d'interprétation. Hal et l'hôtesse réclament du sens ("What thing? ", "What beast? ", "Why an otter? ») aux injures que Falstaff émet comme des énigmes. C'est parce que l'hôtesse prend les mots de Falstaff au sérieux qu'elle veut en connaitre le sens afin de pouvoir les démentir. Dans cette scène, l'injure est et n'est pas un jeu. Cette ambiguïté déjà présente

\footnotetext{
25 « Has not the boy profited ? » commente Hal (2H4, II.ii.81) : ce jeu de l'injure s'apprend.

${ }^{26}$ Roger Caillois, Les Jeux et les hommes. Le masque et le vertige, édition revue et augmentée, Paris, Gallimard, 1967 [1958].
} 
dans la première partie ${ }^{27}$ devient encore plus explicite dans la deuxième partie d'Hen ry IV où Shakespeare brouille davantage encore la frontière entre injure ludique et non ludique.

«Shall we fall foul for toys?» (2H4, II.iv.166)

Dans la deuxième partie d'Henry $I V$, les jeux de l'injure dégénèrent de deux manières différentes : ils tournent à la querelle et à la calomnie. Le monde de la taverne y résonne toujours d'injures de toutes sortes qui deviennent, dans la bouche de Doll notamment, des mots d'affection. Mais il semble désormais impossible de «tourner tout en plaisanterie » («turn all to a merriment », 2H4, II.iv.296). Il semble que les injures sont ici condamnées à avoir un effet de "gravité » (" effect of gravity », $2 \mathrm{H} 4$, I.II.160) que même le jeu de mots de Falstaff sur « gravy» $(2 H 4$, I.ii.161) ne parvient pas à faire oublier.

Les jeux de l'injure sont tout d'abord mis en danger par l'apparition d'un personnage au nom évocateur, Pistol. Ce querelleur ( « swaggerer») est présenté par Doll comme celui qui vient perturber les jeux de la taverne. Décrit comme un trouble-fête, Pistol incarne le risque que le jeu de l'injure a de «mal tourner» («fall foul », $2 \mathrm{H} 4$, II.iv.166). Doll le décrit comme «le gredin le plus mal embouché d'Angleterre ${ }^{28}$ », «the foul-mouth'dst rogue in England» $(2 \mathrm{H} 4$, II.iv.70), ce qui n'est pas sans ironie lorsque l'on connait le langage peu châtié qui règne à la taverne. Ce que Doll et l'hôtesse rejettent en bannissant Pistol, c'est le risque que ces jeux de langue tournent à la querelle («this will grow to a brawl», 2H4, II.iv.168) et à l'affrontement physique. «Are you not hurt i' th' groin ? Methought a made a shrewd thrust at your belly » (2H4, II.iv.206-7) : le jeu d'injure n'admet pas le passage à l'acte et l'anarchie que représente le querelleur. Il s'agit dans cette scène d'exclure le mauvais joueur, celui qui ne respecte pas le pacte de non agression.

\footnotetext{
27 Voir notamment l'ambiguité du fameux « I do ; I will » $(1 H 4$, II.iv.468) et la portée de la question que Lady Percy pose à son mari : « Nay, tell me if you speak in jest or no », $(1 \mathrm{H} 4$, II.iii.95).

${ }^{28}$ Nous utilisons ici la traduction de François-Victor Hugo, Shakespeare, Euvres Complètes, La Pléiade, Paris, Gallimard, 1959.
} 
Une deuxième règle du jeu de l'injure est transgressée dans cette scène : la règle du face à face. Pour qu'il y ait joute ludique il faut que les partenaires soient face à face. Si ce n'est pas le cas, l'injure dégénère en calomnie et le mot qui devrait être sans effet produit « l'effet injure ». Falstaff transgresse cette règle du face-à-face lorsqu'il dit du mal du Prince Hal et de Poins sans savoir que ces derniers l'écoutent :

PRINCE. You whoreson candle-mine you, how vilely did you speak of me even now, before this honest, virtuous, civil gentlewoman ! [...]

FALSTAFF. Didst thou hear me?

$[\ldots]$

PRINCE. I shall drive you then to confess the wilful abuse, and then I know how to handle you.

FALSTAFF. No abuse, Hal, o' mine honour, no abuse.

PRINCE. Not ? - to dispraise me, and call me pantler, and bread-chipper, and I know not what?

FALSTAFF. No abuse, Hal.

PoINS. No abuse?

FALSTAFF. No abuse, Ned, i' th' world, honest Ned, none.

(2H4, II.iv.297-315)

Dans la deuxième partie d'Henry $I V$, il ne va plus de soi que les injures sont inoffensives («no abuse»). Ce que Falstaff nie ici, c'est "l'effet injure » que ses mots peuvent avoir. Mais en niant l'injure, il la met en valeur. Le «no abuse » semble ici inefficace. Si l'on peut à la limite réglementer la langue, il est en revanche impossible de contrôler l'oreille de l'autre. L'explication de texte que Falstaff propose à Hal en lui disant qu'il a dit du mal de lui afin de faire le bien ( «I have done the part of a careful friend and a true subject », 2H4, II.iv.318-19) est censée faire oublier l'effet perlocutoire de ses propos calomnieux. Si la plaisanterie parvient à amortir l'injure, elle ne réussit cependant pas à l'effacer. Les mots d'injure ont du mal à se métamorphoser en mots d'amour et d'amitié. Dans un ouvrage intitulé Mirth Making, The Rhetorical Discourse on Jesting in Early Modern England, Chris Holcomb ${ }^{29}$ présente la plaisanterie comme une activité risquée qui peut toujours mal tourner, être mal reçue ou interprétée : « Jesting [...]

\footnotetext{
${ }^{29}$ Chris Holcomb, Mirth Making. The Rhetorical Discourse on Jesting in Early Modern England, Columbia, University of South Carolina Press, 2001.
} 
is always a flirtation with disorder ${ }^{30}$ ", « Jesting is risky and potentially irreverent business ${ }^{31} »$. La plaisanterie court toujours le risque d'échouer, de ne pas être «heureuse » ou appréciée par l'auditoire. Plaisanter est un jeu dangereux. Holcomb voit dans cet épisode de l'acte II, scène iv, l'échec d'une plaisanterie. Falstaff tente de déguiser l'injure en blague mais, selon lui, n'y parvient pas. La source de cet échec est, selon Holcomb, l'inégalité sociale qui sépare les deux hommes : «[it] illustrates how inequalities in social status can be an insurmountable barrier in jesting exchanges ${ }^{32} »$. Si dans la première partie le Prince jouait le jeu de l'injure, dans la deuxième partie le futur Henriv se met hors-jeu. Ses échanges avec Falstaff sont d'ailleurs beaucoup moins nombreux. C'est désormais avec le «Chief Justice » que Falstaff se livre à des joutes verbales. Or le rôle du Grand Juge consiste non pas à jouer le jeu mais à le prohiber. "How now, Sir John? What are you brawling here? » (2H4, II.i.63) : ce qui dans la première partie est perçu comme un jeu devient ici un crime ou un délit. La « tempête d'imprécations » qui était source d'amusement dans 1 Henry IV est déclarée hors-jeu: "Fie! what man of good temper would endure this tempest of exclamation? » $(2 \mathrm{H} 4$, II.i.78-80) ; " It is not $[. .$.$] the throng of words that come with such more than impudent$ sauciness from you, can thrust me from a level consideration " (II.i.109-112). Le Grand Juge fait office de contrôleur des langues. En pardonnant au Juge ( $2 \mathrm{H} 4$, v.ii), le roi Henri v adopte sa politique de la langue. En bannissant Falstaff, c'est bien, comme l'avait annoncé Warwick, une langue grossière ("gross terms", 2H4, Iv.iv.73) qu'il rejette. Ceux qui refusent de prendre part à ces jeux de la langue les déclarent des péchés. En matière de jeux de langue, l'heure est à la prohibition. Falstaff incarne à lui seul tous les péchés de la langue tels que les définissent les traités religieux médiévaux. Il est un condensé de toutes les mauvaises langues. Au XII ${ }^{\mathrm{e}}$ siècle, Saint Bernard par exemple énumère cinq types de langue à bannir : la langue dissolue (porteuse de paroles futiles), la langue impudique (porteuse de paroles

\footnotetext{
30 Ibid., p. 3 et p. 27.

${ }^{31}$ Ibid., p. 37.

${ }^{32}$ Ibid., p. 175 .
} 
lascives), la langue grandiloquente (porteuse de paroles arrogantes), la langue trompeuse (porteuse de mensonges et de flatteries), la langue médisante (porteuse d'injures et de diffamations) ${ }^{33}$. Falstaff semble incarner toutes ces langues à la fois. Il est également l'épitomé du lien que ces textes monastiques construisent entre gourmandise et loquacité. Dans Les Péchés de la Langue. Discipline et éthique de la parole dans la culture médiévale, Carla Casagrande et Silvana Vecchio rappellent le lien qui s'établit entre les deux fonctions de la langue :

[...] cet organe qui préside aux deux fonctions du parler et du manger est évidemment l'élément qui unifie aussi les deux catégories de péchés qu'on commet avec la langue. Le péché in loquela est inextricablement lié au péché in gustu. [...] goût et parole procèdent du même organe et [...] souvent le désordre de l'un va de pair avec celui de l'autre. [...] au fur et à mesure que le ventre se remplit, observe Grégoire, la langue ne connaît plus de frein ${ }^{34}$.

À la lumière de ces considérations, les mots du roi, lorsqu'il bannit Falstaff, prennent tout leur sens: «Leave gormandizing » $(2 \mathrm{H} 4$, v.v.53). Casagrande et Vecchio notent que, dans un traité d'Oxford du $\mathrm{XII}^{\mathrm{e}}$ siècle (De Lingua), le péché de gula (gourmandise) est défini comme l'ensemble des deux grands domaines, celui du goût (gustus) et celui de la parole (locutio), « retrouvant ainsi un vieux lien entre table et parole affirmé par le christianisme des premiers siècles et le monachisme du haut Moyen Âge 35 ». Le roi demande à son sujet de contrôler sa langue. En quittant le jeu, Henri v réduit Falstaff à ce statut de langue intempérante. La mise à l'index de ces jeux de langue est confirmée lorsque Lancaster conclut : «But all are banish'd till their conversations / Appear more wise and modest to the world " $(2 \mathrm{H} 4$, v.v.100-101). La formulation adoptée par Lancaster («till their conversation appear more wise and modest to the world.») est

\footnotetext{
33 Voir Carla Casagrande et Silvana Vecchio Les Péchés de la Langue. Discipline et éthique de la parole dans la culture médiévale, traduit le l'italien par Philippe Baillet, Les Éditions du Cerf, Paris, 1991, p. 23-24.

34 Op. cit., p. 114-15. Voir aussi p. 123: «Si la nature a donné à l'homme un seul organe pour deux fonctions aussi importantes que le parler et le manger; si, en outre, elle a voulu que cet organe soit particulièrement petit, c'est parce qu'elle a entendu montrer, dès le plan anatomique, la nécessité que l'homme en use avec modération et parcimonie dans les deux activités. La sobriété envers la nourriture et les paroles est inscrite dans l'ordre même de la nature et comme telle, représente une loi morale valable pour tous les hommes. »

35 Op. cit., p. 14.
} 
révélatrice : ce qui a changé, c'est la réception de ces jeux de langue. C'est l'oreille du Prince qui a changé et qui ne reçoit plus les mots de Falstaff de la même façon : "Reply not to me with a fool-born jest ; / Presume not that I am the thing I was. » (v.v.55-6). Hal a fini de jouer ce jeu-là ; il n'est plus d'humeur à jouer ce jeu-là. « God save thy Grace, King Hal, my royal Hal! », "God save thee, my sweet boy! », "My King! My Jove! I speak to thee, my heart!» (2H4, v.v.41-6) : ironiquement, c'est lorsque Falstaff se met à dire à Hal des mots doux que le Roi le bannit. Falstaff, quant à lui, s'évertue à déguiser en jeu l'outrage que le roi vient de lui infliger : " This that you heard was but a colour » $(2 \mathrm{H} 4$, v.v.85-86). La formulation («this that you heard ») là encore est révélatrice : Falstaff se refuse à entendre les paroles du roi, se refuse à leur accorder « l'effet injure ». C'est « pour rire » nous dit-il, « c'est pour du semblant». C'est "No abuse». Le début d'Henry V annoncé ici par le jeu de mots de Shallow ( A colour that I fear you will die in, Sir John », $2 H 4$, v.v.87) fait apparaître l'échec de cette dernière acrobatie verbale.

Ainsi, après avoir mis en scène tous les jeux de l'injure sans effet, Shakespeare nous montre à la fin d'Hen ry IV l'effet le plus extrême que peut avoir l'injure. C'est sur une scène d'injure que se ferme la pièce. S'ils n'en ont pas la forme, les mots du Roi en ont diablement l'effet. On mesure ici encore tout le «jeu », toute l'ambiguité d'une langue qui n'émet que du vent mais peut aussi tuer. En recourant à son sport favori qu'est la plaisanterie, Falstaff ne parvient qu'à amortir provisoirement la violence que lui inflige le roi. Les paroles du roi sont telles que même ce glouton de Falstaff ne peut les digérer. Ces mots lui resteront en travers de la gorge, en travers du gosier. C'est un Falstaff meurtri qui quitte la scène :

FALSTAFF. My lord, my lord, -

CHIEF JUSTICE. I cannot now speak : I will hear you soon.

(2H4, v.v.93-4)

Falstaff a mutilé Hotspur. Henri et le Grand Juge le mutilent à leur tour en lui coupant la langue. Lorsque Lancaster conclut la pièce en disant «I heard a bird so sing, / Whose music, to my thinking, pleas'd 
the King. Come, will you hence»? » (2H4, v.v.107-9), on comprend qu'Henri exclut dorénavant le charivari falstaffien pour lui préférer une musique moins discordante.

Nathalie VIENNE-GUERRIN Université de Rouen 